\title{
RF VOLTAGE MODULATION AT DISCRETE FREQUENCIES, WITH APPLICATIONS TO CRYSTAL CHANNELING EXTRACTION
}

\author{
W. GABELLA ${ }^{1}$, J. ROSENZWEIG $^{1}$, R. KICK ${ }^{2}$ and S. PEGGS ${ }^{3}$ \\ ${ }^{1}$ Physics Department, University of California, Los Angeles \\ ${ }^{2}$ Illinois Mathematics and Science Academy, Aurora, Illinois \\ ${ }^{3}$ Fermilab, Batavia, Illinois
}

(Received 23 May 1992; in final form 7 August 1993)

\begin{abstract}
$\mathrm{Rf}$ voltage modulation at a finite number of discrete frequencies is described in a Hamiltonian resonance framework. The theory is applied to the problem of parasitic extraction of a fixed-target beam from a highenergy proton collider, using a bent crystal as a thin "septum" with an effective width of about one $\mu \mathrm{m}$. Three modes of employment of discrete resonances are proposed. First, a single, relatively strong, static "drive" resonance may be used to excite a test proton so that it will penetrate deeply into the channeling crystal. Second, a moderately strong "feed" resonance with a ramped modulation tune may be used to adiabatically trap protons near the edge of the beam core and transport them to the drive resonance. Third, several weak resonances may be overlapped to create a chaotic amplitude band, either to transport protons to the drive resonance, or to provide a "pulse stretching" buffer between a feed resonance and the drive resonance. Extraction efficiency is semi-quantitatively described in terms of characteristic "penetration," "depletion," and "repetition" times. Simulations are used to quantitatively confirm the fundamental results of the theory, and to show that a prototypical extraction scheme using all three modes promises good extraction performance.
\end{abstract}

KEY WORDS: Extraction, Beam Transport, Magnets: Septum, Particle Dynamics

\section{INTRODUCTION}

This paper describes aspects of the theory of discrete frequency rf voltage modulation in a hadron synchrotron. Reference is frequently made to the problem of slow extraction from a proton storage ring onto a crystal channeling "septum" inside the vacuum chamber. According to whether or not the reader is interested in this novel technique, he or she may view these extraction discussions either as a primary motivation or as a continuing illustrative example. In either case, a brief introduction to the crystal extraction problem is appropriate.

The Supercollider Fixed Target experiment (SFT) proposes to extract fixed-target beam parasitically from the Superconducting Super Collider (SSC) while colliding beam experiments are also in operation ${ }^{1,2,3}$. Although it is impossible to be completely 
TABLE 1: Nominal parameters at the crystal for a Tevatron test of channeling extraction using rf voltage modulation, and for a preliminary conceptual SSC design.

\begin{tabular}{lccc}
\hline Property & Units & Tevatron test & SSC concept \\
\hline Momentum, $p_{0}$ & TeV/c & 0.9 & 20.0 \\
RMS $\Delta p / p_{0}, \sigma_{\delta}$ & & $0.2 \times 10^{-3}$ & $0.05 \times 10^{-3}$ \\
Separatrix $\Delta p / p_{0}, \delta_{\text {sep }}$ & & $0.6 \times 10^{-3}$ & $0.25 \times 10^{-3}$ \\
Dispersion, $\eta_{x}$ & $\mathrm{~m}$ & 2.9 & 4.0 \\
Bucket width, $\eta_{x} \delta_{\text {sep }}$ & $\mathrm{mm}$ & 1.75 & 1.0 \\
Synchrotron tune, $Q_{s 0}$ & & 0.00089 & 0.00126 \\
Synchrotron period, $T_{s}$ & $\mathrm{turns}$ & 1127 & 791 \\
Normalized emittance $(1 \sigma)$ & $\mu \mathrm{m}$ & 3.0 & 1.0 \\
Beta function, $\beta_{x}$ & $\mathrm{~m}$ & 98 & 1320 \\
Betatron size, $\sigma_{x}$ & $\mathrm{~mm}$ & 0.55 & 0.25 \\
Drive modulation depth, $\varepsilon$ & & 0.01 & 0.01 \\
\hline
\end{tabular}

parasitic, as is discussed below, it is not permissible to set the betatron tunes to arbitrary values, since this would disrupt collision conditions. Conventional slow extraction schemes, ${ }^{4}$ using a horizontal tune close to $\frac{1}{2}$ or $\frac{1}{3}$, are therefore not viable. Because of this, most attention has turned to manipulations of the radiofrequency (RF) system that controls longitudinal dynamics. Various types of rf manipulation have been proposed, including the excitation of amplitude modulations at discrete frequencies, ${ }^{5}$ as well as phase modulation excitation. Assorted noise distributions have been discussed, including wideband, narrowband, and a notched noise spectrum ${ }^{6,7,8,9}$.

All of the rf manipulation schemes have the same general arrangement. There is a crystal close to the beam at a position in the ring where the horizontal dispersion function $\eta_{x}$ is large. The betatron motion may be temporarily ignored, with some justification, if the horizontal beta function $\beta_{x}$ is relatively small. A test proton in the rf bucket strikes the crystal if its synchrotron displacement is larger than the crystal displacement. Suppose that a particular proton just misses the crystal at the extreme displacement of one synchrotron oscillation, but has a large enough amplitude one synchrotron period later, at its next extremum, to strike the crystal. This proton is efficiently channeled and extracted if it hits sufficiently deep within the crystal. Ideally, all protons would enter the crystal at least ten times deeper than the accuracy with which the crystal surface can be polished. This results in the nominal (somewhat conservative) requirement that most protons should strike the crystal at a depth of greater than $1 \mu \mathrm{m}$.

Naively, then, a successful rf manipulation scheme must increase the synchrotron oscillation amplitude of a test proton near the crystal by at least $1 \mu \mathrm{m}$ in one synchrotron period. All of the proposed schemes provide a mechanism by which 
appropriate test protons spiral outward with an increasing oscillation amplitude. This commonality makes it possible to include betatron oscillations in a realistic analysis (below) that is independent of the details of the excitation mechanism. Noise excitation is not discussed any further here, except in the context of the overlap of a small number of amplitude modulation resonances. Phase modulation is not discussed at all, in order to concentrate on discrete-frequency amplitude-modulation schemes. These schemes keep the stable fixed point center of the rf bucket at a constant location, and are therefore much less prone to dilution of the phase-space density of the beam core than phase modulation schemes.

Table 1 lists a set of nominal parameters that apply to a proposed test of crystal channeling extraction in the Tevatron ${ }^{10}$ and a set corresponding to a rough conceptual design for the actual SSC extraction scheme. The nominal Tevatron numbers are used below in the evaluation of various quantities for the proposed test. However, it should be recognized that the actual conditions of crystal extraction could be quite different - especially in the SSC configuration, but also in a Tevatron test.

\section{UNPERTURBED MOTION}

The unperturbed longitudinal motion system is parameterized by two numbers $-Q_{s 0}$, the small oscillation synchrotron tune, and $\delta_{\text {sep }}=(\Delta p / p)_{\text {sep }}$, the value of the offmomentum parameter at the separatrix of the rf bucket. For example, if $Q_{s 0} \ll 1$, the motion of the rf phase $\phi$ in a storage ring is approximated well by

$$
\frac{d^{2} \phi}{d t^{2}}+\left(2 \pi Q_{s 0}\right)^{2} \sin (\phi)=0,
$$

where time, $t$, is measured in units of accelerator turns. This is also the equation of motion of a pendulum. It is convenient to immediately write down a Hamiltonian that describes this motion,

$$
H=\left(2 \pi Q_{s 0}\right)\left[\frac{1}{2} p^{2}+(1-\cos (\phi))\right]
$$

The canonical equations of motion of this system are

$$
\begin{aligned}
& \frac{d p}{d t} \equiv-\frac{\partial H}{\partial \phi}=-\left(2 \pi Q_{s 0}\right) \sin (\phi) \\
& \frac{d \phi}{d t} \equiv \frac{\partial H}{\partial p}=\left(2 \pi Q_{s 0}\right) p
\end{aligned}
$$

After differentiating Equation ( $3 \mathrm{~b}$ ) with respect to time, and substituting the right hand side of Equation (3a) for $\frac{d p}{d t}$, the equation of motion, Equation (1), is recovered.

Pursuing the pendulum analogy, if $\phi$ represents the angle from the vertical, then $p$ represents the (scaled) angular velocity. The separatrix of the system is drawn by 
tracing the curve $H=4 \pi Q_{s 0}$, corresponding to the energy of the "pendulum" at rest $(p=0)$ at its unstable equilibrium position $(\phi=\pi)$. The largest values of $p$ on the separatrix occur when the pendulum passes through the vertical $(\phi=0)$, giving $|p|_{\max }=2$. This justifies identifying

$$
p \equiv 2 \frac{\delta}{\delta_{\text {sep }}}
$$

as the normalized off-momentum parameter. If the angle $\phi$ is always much smaller than unity, then the Hamiltonian can be written approximately as

$$
H \approx\left(2 \pi Q_{s 0}\right) \frac{1}{2}\left[p^{2}+\phi^{2}\right] .
$$

Since the value of the Hamiltonian is conserved along any single trajectory, the quantity in square brackets is also conserved, showing that a small amplitude trajectory describes a circle in the $(p, \phi)$ phase space.

It is convenient to introduce the oscillation amplitude $a$, where

$$
2 a^{2} \equiv \frac{1}{2} p^{2}+(1-\cos (\phi))
$$

for small or large oscillations. Since the Hamiltonian given in Equation (2) is simply proportional to the square of the amplitude, the amplitude is constant along a trajectory. The oscillation amplitude may also be defined in terms of the off-momentum parameter through

$$
a=\frac{\delta_{\max }}{\delta_{\mathrm{sep}}},
$$

where $\delta_{\max }$ is the largest off-momentum parameter encountered in a given trajectory. Normalization of the amplitude is such that it has a value of 1 at the separatrix.

Figure 1 plots the Poincaré surface of section for the simulated longitudinal motion of protons in an rf bucket. Several protons with different amplitudes are launched, with one dot plotted per turn around an accelerator - the Poincaré period. In this example the synchrotron tune is $Q_{s 0}=0.0089$, ten times the nominal Tevatron value, to make the discrete nature of the curves apparent. Motion is propagated by iterating a map of two successive difference equations, not by integrating a differential equation.

\section{CHANGE OF TUNE WITH AMPLITUDE}

The shape of a trajectory is reasonably circular when $a \ll 1$, in which case the polar coordinates $a$ and $\theta$ shown in Figure 1 are natural. For small motion, then,

$$
\begin{aligned}
& \frac{p}{2}=\frac{\delta}{\delta_{\mathrm{sep}}} \approx-a \sin (\theta), \\
& \frac{\phi}{2} \quad \approx a \cos (\theta),
\end{aligned}
$$




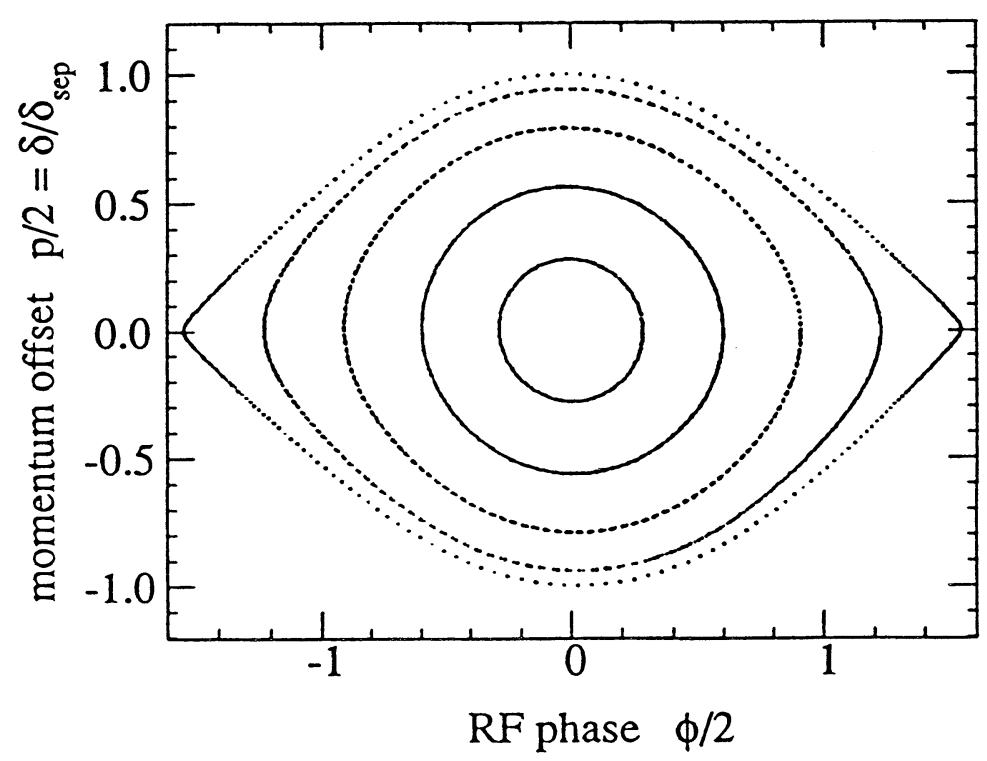

FIGURE 1: Poincaré surface of section for simulated longitudinal motion. Each dot represents one turn of a trajectory in the phase space of the rf phase and the off-momentum parameter. In order to show individual points, a synchrotron tune of ten times the nominal Tevatron value was used.

and

$$
\theta \approx 2 \pi Q_{s 0} t
$$

As the oscillation amplitude is increased, the trajectories become less circular, and the synchrotron tune $Q_{s}$ decreases. This detuning is calculated, to first order in $a$, by rewriting Equation (3a) after expanding $\sin (\phi)$ to cubic order. The right-hand side then contains a linear part and a nonlinear perturbation $\Delta p_{n l}$, which is written for a finite time step $\Delta t$ as

$$
\Delta p_{n l}=\frac{\pi Q_{s 0}}{3} \phi^{3} \Delta t
$$

This is illustrated in Figure 2. Since the corresponding nonlinear phase advance is

$$
\Delta \theta_{n l}=-\frac{\cos (\theta)}{2 a} \Delta p_{n l}
$$

the perturbed tune is simply

$$
Q_{s}(a)=Q_{s 0}+\frac{1}{2 \pi}\left\langle\frac{d \theta_{n l}}{d t}\right\rangle \approx Q_{s 0}\left[1-\frac{2}{3} a^{2}\left\langle\cos ^{4}(\theta)\right\rangle\right],
$$



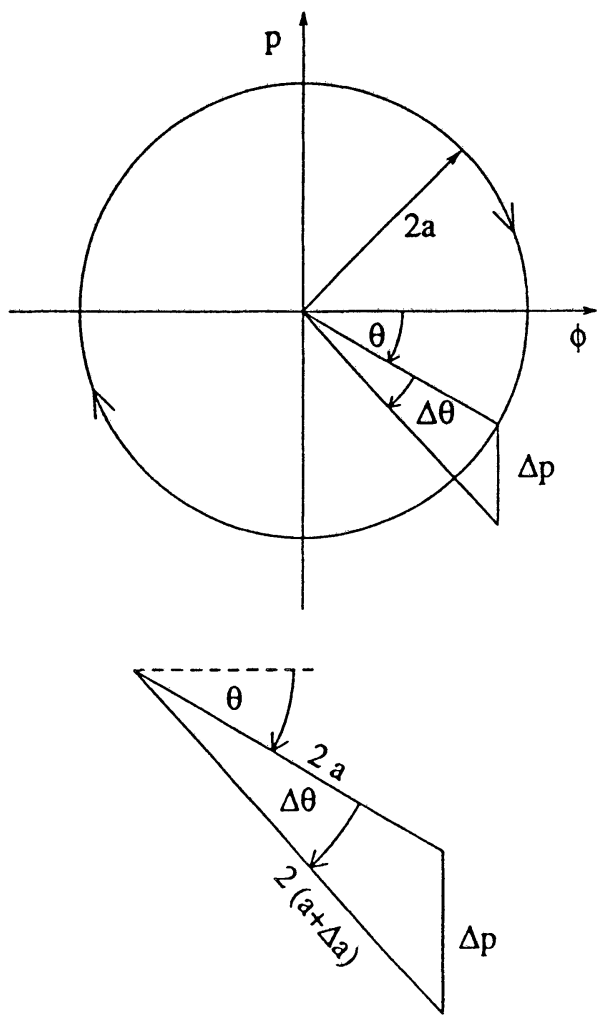

FIGURE 2: The effect of a momentum kick on the angular coordinate of the trajectory.

where angle brackets signify an average over time. This gives

$$
Q_{s} \approx Q_{s 0}\left(1-\frac{1}{4} a^{2}\right)
$$

In the limit that the difference equations are approximated well by differential equations, $Q_{s 0} \ll 1$, the motion can be solved exactly, giving the dependence of tune upon amplitude as

$$
\begin{gathered}
Q_{s}=\frac{\pi Q_{s 0}}{2 K\left(a^{2}\right)} \approx Q_{s 0}\left(1-\frac{1}{4} a^{2}-\frac{5}{64} a^{4} \ldots\right), \\
K(m)=\frac{\pi}{2} \sum_{j=0}^{\infty}\left[\frac{(2 j-1) ! !}{(2 j) ! !}\right]^{2} m^{j}
\end{gathered}
$$




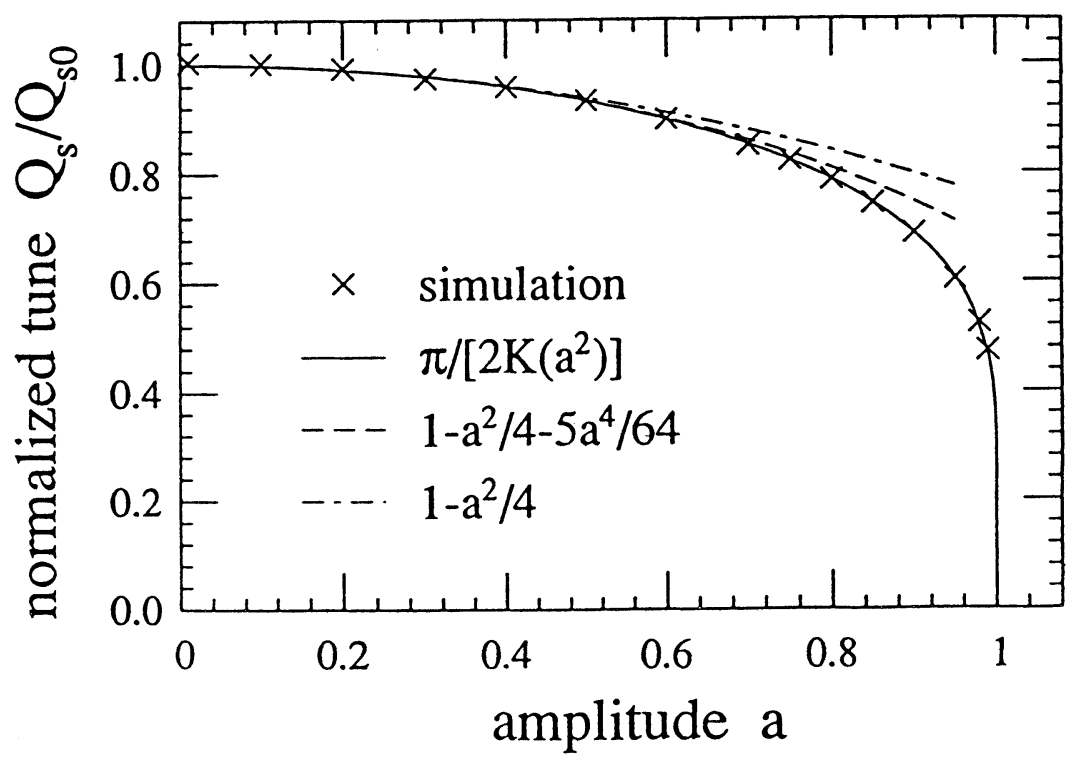

FIGURE 3: The variation of synchrotron tune with amplitude in the rf bucket. Various analytic approximations mentioned in the text are compared with simulation results.

where $K(m)$ is a complete elliptical integral of the first kind. Figure 3 compares how well Equation (13) and (14) agree with the variation of tune versus amplitude found by a simple simulation. Even the lowest-order expansion is accurate up to surprisingly large amplitudes.

\section{SPIRAL MOTION DUE TO AMPLITUDE MODULATION}

When the rf voltage is modulated to a small relative depth of $\varepsilon \ll 1$ at a single tune $Q_{m}$, the equation of motion, Equation (3a), becomes explicitly time dependent

$$
\frac{d p}{d t}=-2 \pi Q_{s 0}\left[1+\varepsilon \cos \left(2 \pi Q_{m} t+\alpha\right)\right] \sin (\phi)
$$

Here $\alpha$ is the phase of the modulation at time $t=0$. In order to calculate the effect of this modulation at small amplitudes, the effect of the perturbation over a small time step $\Delta t$ is again separated out. This gives, to first order in $\phi$,

$$
\Delta p_{m}=-\varepsilon 2 \pi Q_{s 0} \cos \left(2 \pi Q_{m} t+\alpha\right) \phi \Delta t
$$


Assuming that the unperturbed motion is given by Equation (8), the effect of this perturbation is to change the amplitude of the motion by

$$
\begin{aligned}
\Delta a & =-\frac{\Delta p_{m}}{2} \sin (\theta) \\
& =\varepsilon a \frac{\pi}{2} Q_{s 0}\left[\sin \left(2 \pi\left\{2 Q_{s}+Q_{m}\right\} t+\alpha\right)+\sin \left(2 \pi\left\{2 Q_{s}-Q_{m}\right\} t-\alpha\right)\right] \Delta t,
\end{aligned}
$$

where the base tune $Q_{s 0}$ in Equation (9) has been replaced by the modified tune $Q_{s}$, and trigonometric identities have been applied. The second term inside the square brackets is slowly varying if $Q_{m} \approx 2 Q_{s}$, in which case the average rate of amplitude increase is

$$
\frac{d a}{d t}=-\varepsilon a \frac{\pi}{2} Q_{s 0} \sin (\alpha)
$$

for moderate times $|t| \ll 1 /\left|2 Q_{s}-Q_{m}\right|$. This causes the motion to be modified from a circle to a spiral, with a gain factor per synchrotron period of $T_{s}$ turns of approximately

$$
g \equiv \frac{\Delta a}{a}=-\varepsilon \frac{\pi}{2} \sin (\alpha) .
$$

In other words, in one turn of the spiral (one synchrotron period) the amplitude increases by an amount of the same order as the depth of the amplitude modulation. Using the nominal value for $\varepsilon$ recorded in Table 1, this increase is as large as $1.57 \%$ per period.

\section{RESONANCE ISLANDS WITHIN THE RF BUCKET}

Figure 4 illustrates how amplitude modulation creates two resonance islands inside the rf bucket. One dot is plotted in this figure per modulation period of 605 turns, which is now the Poincaré period in which the difference equations repeat themselves exactly. Similar plots for different modulation phase $\alpha$ would show islands that have "rotated" inside the rf bucket. A proton responds resonantly to the amplitude modulation if its synchrotron tune $Q_{s}(a)$ is approximately half of $Q_{m}$, the modulation tune. Stable fixed points at the center of the resonance islands are located at a resonance amplitude $a_{R}$ given by

$$
Q_{s}\left(a_{R}\right)=\frac{1}{2} Q_{m}
$$

which is approximately solved by inverting Equation (13) to give

$$
a_{R}=2\left(1-\frac{Q_{m}}{2 Q_{s 0}}\right)^{1 / 2}
$$




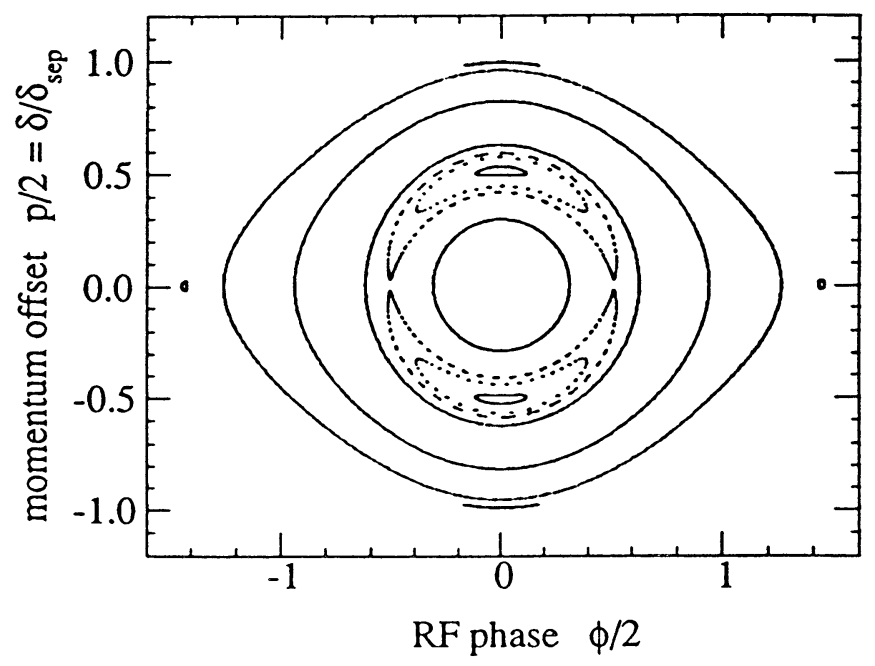

FIGURE 4: Poincaré surface of section for simulated longitudinal motion with amplitude modulation. The points are plotted every modulation period of 605 turns. The ratio $Q_{m} / Q_{s 0}$ is approximately 1.864 , so the center of the resonance island, where $Q_{m} / Q_{s}(a)=2$, is located at an amplitude $a=0.5$.

for moderate amplitudes. Technically, there is a family of Mathieu resonances at amplitudes with synchrotron tunes equal to $\frac{k}{2} Q_{m}$ where $k$ is an integer ${ }^{11}$, but in practice only the $k=1$ fundamental resonance has significant strength.

Motion under the influence of rf voltage modulation, described by Equations ( $3 b$ ) and (15), is summarized by the Hamiltonian

$$
H=\left(2 \pi Q_{s 0}\right)\left[\frac{1}{2} p^{2}+\left\{1+\varepsilon \cos \left(2 \pi Q_{m} t+\alpha\right)\right\}\{1-\cos (\phi)\}\right]
$$

It is useful to perform a canonical transformation to action angle variables, $(J, \Psi)$, in order to conveniently describe the motion. Old and new coordinates are related by

$$
\begin{aligned}
& p=-(2 J)^{1 / 2} \sin (\Psi), \\
& \phi=(2 J)^{1 / 2} \cos (\Psi) .
\end{aligned}
$$

The transformed Hamiltonian becomes

$$
H=\left(2 \pi Q_{s 0}\right)\left[J-\frac{J^{2}}{6} \cos ^{4} \Psi+\varepsilon J \cos \left(2 \pi Q_{m} t+\alpha\right) \cos ^{2} \Psi\right] .
$$


Terms of order $\varepsilon J^{2}, J^{4}$ and higher are dropped here and in subsequent expressions. For small amplitudes close to the center of the rf bucket, the action $J$ is a constant of the motion, while the angle $\Psi$ advances smoothly in time. That is,

$$
\begin{aligned}
& J \approx 2 a^{2}, \\
& \Psi \approx 2 \pi Q_{s 0} t .
\end{aligned}
$$

The isolated resonance Hamiltonian is derived by using Equation (25b) and the approximation $Q_{m} \approx 2 Q_{s 0}$, and averaging the motion over one modulation period. Only slowly varying terms survive the averaging process. After these manipulations, the time-averaged Hamiltonian becomes

$$
H=\left(2 \pi Q_{s 0}\right)\left[J-\frac{J^{2}}{16}+\frac{\varepsilon J}{4} \cos \left(2 \Psi+2 \pi Q_{m} t+\alpha\right)\right],
$$

which has a surprisingly simple form.

The description of proton behavior near the modulation islands becomes even simpler after a canonical transformation to the "slow" phase angle

$$
\chi=\Psi-\frac{1}{2}\left(2 \pi Q_{m} t+\alpha\right)
$$

is made, and the Hamiltonian is expanded about the stable fixed point at the island center by using

$$
J=J_{R}+\Delta J,
$$

where $J_{R}$ is called the resonant action. After making these transformations, dropping a higher-order term proportional to $\varepsilon \Delta J$, and arbitrarily adding a constant term, the resonance Hamiltonian becomes

$$
H_{R}=-\frac{\pi Q_{s 0}}{4}\left[\frac{1}{2}(\Delta J)^{2}+2 \varepsilon J_{R}(1-\cos (2 \chi))\right],
$$

which is analogous to the pendulum Hamiltonian of Equation (2). Reduction of the two first-order canonical equations of motion (found by differentiation of $H_{R}$ ) into a single second-order differential equation shows that small-amplitude motion close to the stable fixed point at $(\Delta J, \chi)=(0,0)$ is described by

$$
\chi=\chi_{\max } \cos \left(2 \pi Q_{I} t\right) .
$$

Here the "island tune" $Q_{I}$ is given by

$$
\frac{Q_{I}}{Q_{s 0}}=\varepsilon^{1 / 2} \frac{a_{R}}{2}=\left(\frac{\varepsilon J_{R}}{8}\right)^{1 / 2}
$$




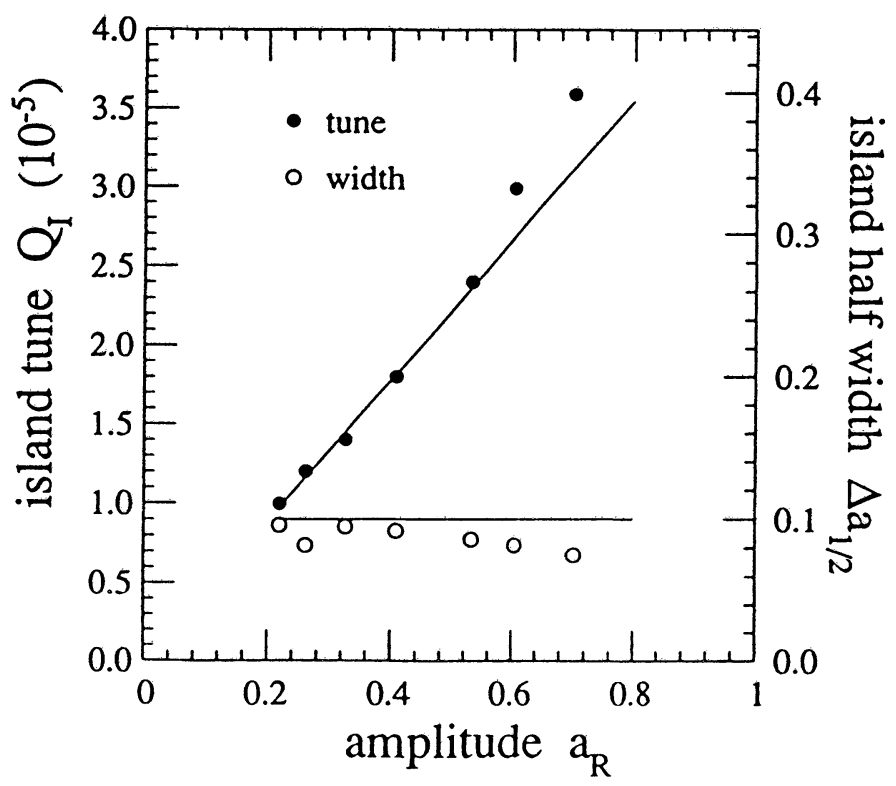

FIGURE 5: Comparison of simulation results with analytic predictions for the island tune and the island half width. Simulation results are shown as filled and open circles for the island tune and for the half-width, respectively. Analytic predictions are shown as straight lines.

and is usually at least an order of magnitude smaller than the synchrotron tune. That is, a proton under the influence of even a strong resonance moves from one side of the island to the other in a time that is long compared to the synchrotron period. The island size is parameterized by the half-width of the separatrix of the resonance, given by

$$
\begin{aligned}
\Delta J_{1 / 2} & =\left(8 \varepsilon J_{R}\right)^{1 / 2}, \\
\Delta a_{1 / 2} & =\varepsilon^{1 / 2} .
\end{aligned}
$$

These results are remarkably simple, especially when expressed in terms of the resonant amplitude. Their accuracy is confirmed by the results from simulation shown in Figure 5. The presence of the square root shows that even very-small-amplitude modulation perturbations have a significant effect.

\section{THREE CHARACTERISTIC EXTRACTION TIMES}

The stage is now set - at last - to derive some practically achievable parameters of a crystal channeling extraction scheme using rf amplitude modulation. Assume for 
now that protons are somehow injected into the low-amplitude side of a drive island that is centered at a large amplitude near the crystal. (The viability and behavior of transport mechanisms that carry protons from near the core of the rf bucket out to the drive island are discussed below.) A good approximation to the total horizontal displacement of a proton is

$$
x_{\text {total }}=a_{\beta} \cos \left(2 \pi Q_{x} t+\phi \beta\right)+a_{s}\left[1+g Q_{s} t\right] \cos \left(2 \pi Q_{s} t\right) .
$$

The gain factor $g \ll 1$ is defined in Equation (19). It varies only slightly in a few synchrotron periods, so treating it as a constant is often possible. Betatron oscillations are parameterized by their amplitude and phase, $a_{\beta}$ and $\phi_{\beta}$, and by $Q_{x}$, the fractional part of the horizontal tune. In real space the synchrotron oscillation amplitude is given by

$$
a_{s}=a \eta_{x} \delta_{\mathrm{sep}}
$$

where a typical value for $a_{s}$, using the nominal Tevatron values in Table 1 and putting $a=0.85$, is $1.5 \mathrm{~mm}$.

What is the typical depth, or step size, of the protons as they strike the crystal? How efficient is the process? Accurate quantitative answers to these questions necessarily rely on detailed numerical simulations, such as those summarized at the end of this paper. However, approximate answers and considerable insight are gained by describing the problem in terms of three characteristic times: the penetration time, the depletion time, and the repetition time. All three times must be approximately equal in order for extraction to be efficient. This description is not limited to an amplitude modulation scheme, but is quite general - it holds for any channeling scheme whose rf perturbation strength is measured by a single parameter $g$, the spiral gain factor.

The test protons of interest lie on a single circle of constant betatron amplitude $a_{\beta}$, and are evenly distributed in betatron phase. This betatron circle just misses the edge of the crystal one synchrotron period before the time origin, at $t=-T_{s}$, so the initial condition is

$$
x_{\text {crystal }}=a_{\beta}+a_{s}[1-g] .
$$

For small times, $|t| \ll T_{s}$, the total displacement of the proton relative to the crystal surface is approximated well by

$$
x_{\text {total }}-x_{\text {crystal }}=a_{\beta}\left[\cos \left(2 \pi Q_{x} t+\phi_{\beta}\right)-1\right]+a_{s}\left[g+\cos \left(2 \pi Q_{s} t\right)-1\right] .
$$

This is illustrated in Figures 6a and 6b, which also show the two angles $\Psi_{s}$ and $\Psi_{\beta}$ that are referred to below.

The possibility of a proton hitting the crystal first exists at the penetration time $t_{p}$ before the time origin, $t=-t_{p}$, where

$$
t_{p}=\frac{\Psi_{s}}{2 \pi Q_{s}}=\frac{\cos ^{-1}(1-g)}{2 \pi Q_{s}} \approx \frac{(2 g)^{1 / 2}}{2 \pi Q_{s}} .
$$




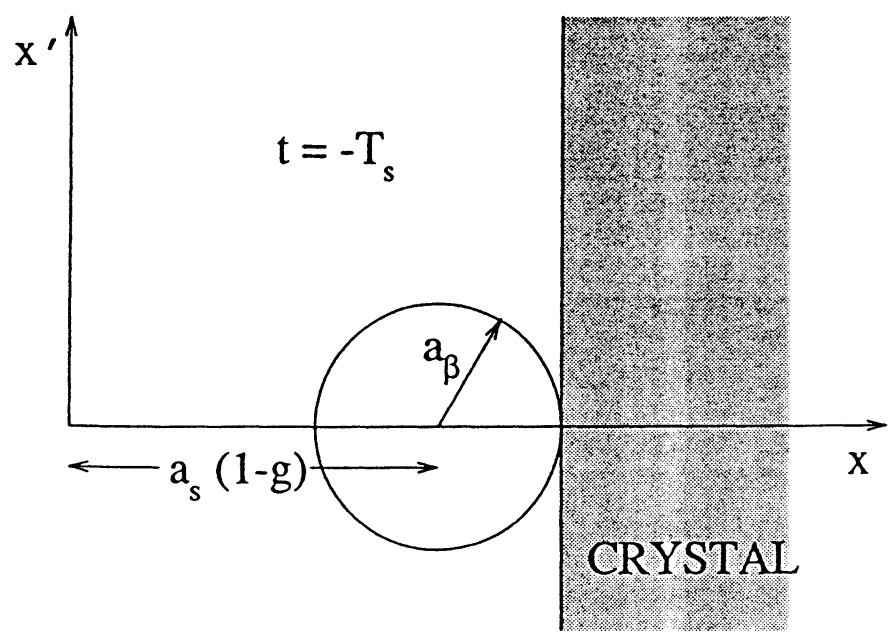

FIGURE 6a: Superposition of synchrotron and betatron motion one synchrotron period $\left(t=-T_{s}\right)$ before protons start to be scraped from the betatron circle.

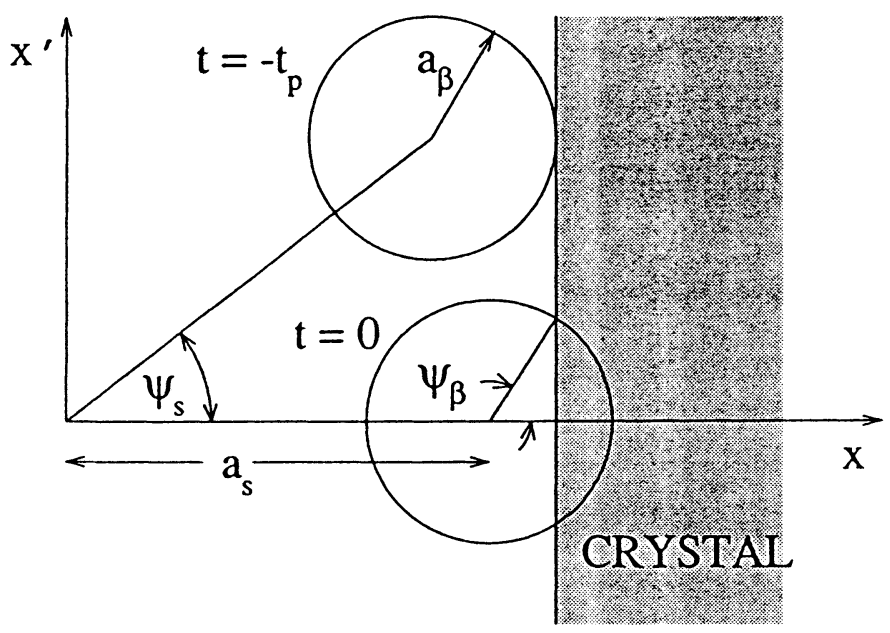

FIGURE 6b: Superposed motion just as the first protons begin to penetrate the extraction crystal $\left(t=-t_{p}\right)$, and when the betatron circle maximally overlaps the crystal $(t=0)$. 
Using the value of $\varepsilon=0.01$ in Table 1 , putting $\sin (\alpha)=-1$, and replacing $Q_{s}$ by $Q_{s 0}$ leads to a nominal penetration time of 32 turns.

Whether or not a proton does in fact hit the crystal on a given turn depends on its betatron phase and on whether or not it has collided on a previous turn. The depletion time $t_{d}$ characterizes the shortest number of turns necessary for all protons on the betatron circle to hit the crystal under conditions of optimum "mixing". The phase distribution of protons rotates by a large angle, $2 \pi Q_{x}$, on each additional turn from $t=-t_{p}$ to $t=0$, while the width of the betatron phase angle bite increases in size up to a maximum of $2 \Psi_{\beta}$. In the naive approximation that all bites are of the maximum size, and that all are non-overlapping, then it takes $t_{d}$ turns for all protons to be scraped, where

$$
t_{d}=\frac{2 \pi}{2 \Psi_{\beta}}=\frac{\pi}{\cos ^{-1}\left(1-g \frac{a_{s}}{a_{\beta}}\right)} \approx \frac{\pi}{(2 g)^{1 / 2}}\left(\frac{a_{\beta}}{a_{s}}\right)^{1 / 2} .
$$

If the proton has a small betatron amplitude, then the depletion time also becomes small, and the extraction efficiency is poor, since the typical penetration depth is proportional to the depletion time. In the extreme case, a zero-amplitude proton has precisely one turn in which to gain enough momentum to jump deeply into the crystal. This is a severe requirement. Fortunately, the bi-Gaussian distribution of protons in betatron space has a maximum probability distribution at $a_{\beta}=\sigma_{x}$, and is vanishingly small as the amplitude approaches zero. Since we desire to maximize the effects of the drive resonance for the most common betatron amplitudes, the crystal should be moved to about $\sigma_{x}$ from the center of the modulation island:

$$
x_{\text {crystal }}=\eta_{x} \delta_{\text {sep }} a_{R}+\sigma_{x} .
$$

Using the values of Table 1 , and replacing $a_{\beta}$ by $\sigma_{x}$, the value of $t_{d}$ becomes 11 turns. This value is a conservative underestimate, since most of the angular bites are significantly smaller than the maximum size - especially the early bites. It is therefore reasonable to expect the real depletion time to be well matched to the nominal penetration time.

The third and final characteristic time is $t_{r}$, the repetition time that characterizes the efficiency of the mixing on the betatron circle. This depends on $Q_{x}$, the betatron tune. For example, if $Q_{x}$ is exactly $\frac{1}{2}$, then every proton regains the same phase after only two turns, and only two bites will ever be taken out of the betatron circle. The protons that are incrementally nibbled from the widening edges of the bites do not penetrate deeply into the crystal. Fortunately, realistic betatron tunes are set to avoid low-order resonances, where $Q_{x} \approx \frac{m}{n}$ with $m$ and $n$ mutually prime integers, in the interests of transverse beam stability. It is not possible to avoid all resonances, because the number line is dense in rational numbers, but it is possible to avoid resonances with denominators up to about 20 , for example. The repetition time $t_{r}$ is simply that denominator, a number well matched to both the nominal penetration time and the nominal depletion time. 
The hits with the deepest penetrations - as deep as $g a_{s} \approx 24 \mu \mathrm{m}$-occur when $t=$ 0 . This is much larger than the required depth size of about $1 \mu \mathrm{m}$, demonstrating that amplitude modulation is a promising mechanism for crystal channeling extraction. In order for these depths to be attainable, the depletion time must be roughly equal to the penetration time, $t_{d} \approx t_{p}$, so that typical protons can survive long enough after the initial, less fortunate protons have begun to strike the crystal. Even the longlived protons will not achieve their full potential depth unless the repetition time is comparable with the depletion time, $t_{r} \approx t_{d}$.

\section{REQUIRED DRIVE ISLAND SIZE}

A final condition must be met if protons with a reasonable range of betatron amplitudes are to be successfully channeled: the full width of the drive island must be at least comparable to the betatron size of the beam. That is, it is necessary that

$$
2 \eta_{x} \delta_{\text {sep }} \Delta a_{1 / 2} \geq \sigma_{x} .
$$

Applying Equation (32b), and using the nominal Tevatron parameters, the required drive modulation strength is

$$
\varepsilon \geq\left(\frac{\sigma_{x}}{2 \eta_{x} \delta_{\text {sep }}}\right)^{2} \approx 0.025 .
$$

This condition is not met by the nominal Tevatron test parameters in Table 1, so crystal extraction will not be efficient for a broad range of betatron amplitudes. Protons with too small an amplitude will not hit the crystal at all, while protons with too large an amplitude will have a small spiral gain factor $g$ and thus a small step size. These effects may be mitigated if one keeps the modulation strength $\varepsilon$ constant, either by decreasing the betatron beam size or by enlarging the physical size of the rf bucket. This concern applies equally to all $\mathrm{rf}$ manipulation techniques to be tested in the Tevatron. This problem is less serious in the SSC, where the requirement $\varepsilon \geq 0.015$ is more modest, and where there is much greater freedom to adjust the optical parameters at the crystal.

\section{ADIABATIC TRAPPING - FEED ISLANDS}

One way to transport protons from the outer edge of the core to the inner edge of the drive island is by carrying them in feed islands whose resonant amplitude slowly increases. This is accomplished by lowering the modulation tune $Q_{m}$ slowly enough so that protons are adiabatically trapped in islands whose widths, etc., are still accurately given by the Hamiltonian expressions derived above. Loosely speaking, the condition for adiabaticity is that the distance the island moves in one island period 

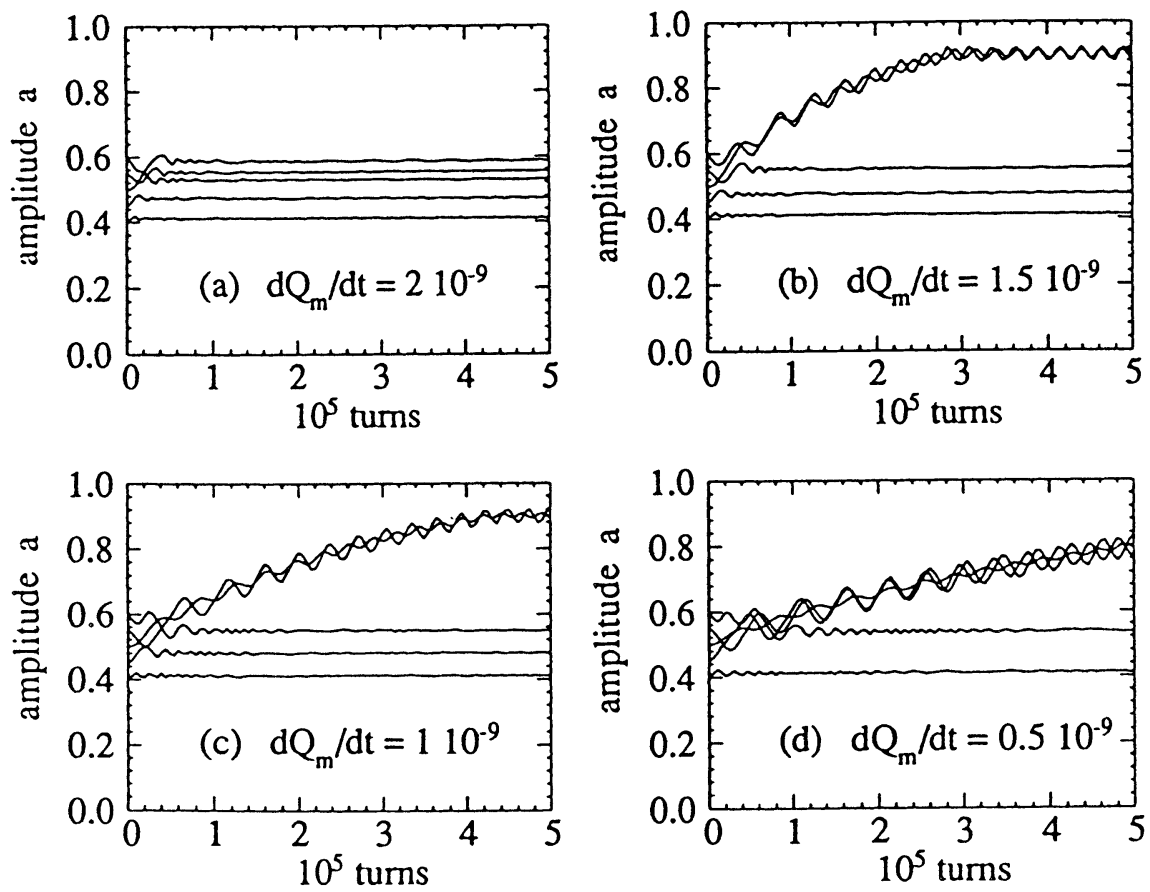

FIGURE 7: The response of protons to moving "feed" islands as the modulation tune ramp rate $\left|d Q_{m} / d t\right|$ is progressively reduced. For adiabatic trapping to occur, the rate of ramping must be less than $1.86 \times 10^{-9}$, according to theory. In each case the same five trajectories are followed, with initial conditions just below, inside, and above the nominal island position.

is smaller than the island half-height. From rigorous Hamiltonian considerations ${ }^{12}$, it can be shown that a stable point at the center of a moving island exists only if

$$
\left|\frac{d Q_{m}}{d t}\right|<2 \pi Q_{I}^{2}=\frac{\pi}{2} \varepsilon a^{2} Q_{s 0}^{2}
$$

When this condition is close to being an equality, the area around the stable point that is capable of successfully trapping protons is greatly reduced in size.

The adiabatic criterion is in good agreement with the numerical simulation tests summarized in Figures $7 \mathrm{a}$ through $7 \mathrm{~d}$, where the rate of change of modulation tune is systematically increased. Since the simulation used $a=0.5$ and the nominal Tevatron values of Table 1, Equation (42) predicts that $1.86 \times 10^{-9}$ is the maximum value for which adiabatic trapping is possible. Each part of Figure 7 shows several protons launched with different initial amplitudes above and below the initial resonance 
amplitude. Protons that are adiabatically trapped are distinguished from untrapped ones by the steady increase in their average amplitude as the center of the feed island moves towards larger amplitudes. Note that $a$ and $Q_{I}$ increase as $Q_{m}$ decreases, so the adiabatic criterion is less stringent at later times.

All of the protons tracked in Figure 7 show many oscillations at a frequency that gradually increases. For trapped particles this merely represents their motion around the moving island center. However, untrapped protons also have oscillations at the beat tune between the modulation tune and their own synchrotron tunes. Untrapped proton oscillation amplitudes decrease as the feed island recedes, but still remain noticeable as the feed island approaches the drive island. Similarly, protons in the sacrosanct core of the beam also respond to rf voltage modulation with a small amplitude oscillation. This does not lead to emittance growth in the core so long as the modulation parameters are constant in time (as with the drive islands), or so long as they change adiabatically slowly (as with the feed islands).

Adiabatically moving resonance islands offer a way to reliably transport large numbers of protons over large distances. In this sense it is (somewhat) analogous to the use of barges in the transportation of bulk raw materials. Unfortunately, the transport is slow - of order 10 seconds in the Figure 7 simulation of Tevatron parameters. Therefore, if a single feed island is used to deliver protons in bulk directly to the drive islands, an undesirable pulsed time structure is imposed on the extracted beam. Fortunately, it is possible to introduce an intermediate transport mechanism between adiabatic islands and drive islands, that acts as a pulse stretcher to smooth out the extraction flux profile.

\section{CHAOTIC ISLAND OVERLAP - PSEUDO-DIFFUSION}

Another voltage modulation transport mechanism uses resonances to create chaotic behavior in a limited range of amplitudes. This section quantitatively describes the creation of such chaotic bands by causing two or more resonances to overlap. Chaos transports protons in much the same way as a noise-based scheme: by relying on the (superficially) diffusive random walk of the protons. In its favor, a chaotic transport process is faster than a noise-based process, is more controllable, and is more localizable in phase space. A chaotic band can be used to transport particles all the way from the beam core to the drive resonance, or it can be used in conjunction with feed islands, as described above.

If an area in phase space is "occupied" by two different resonance islands, then the description of the motion given above is inconsistent. In fact, the Chirikov criterion states (loosely speaking) that the overlap of resonance islands is sufficient to create a region of chaotic orbits in phase space. Assuming that two discrete amplitude modulation perturbations of the same strength $\varepsilon$ are driven at nearly identical modulation tunes $Q_{1}$ and $Q_{2}$, then the islands overlap, and chaos ensues, if

$$
2 \Delta a_{1 / 2}>\left|a_{1}-a_{2}\right| \approx\left|\frac{d a}{d Q_{m}}\left(Q_{1}-Q_{2}\right)\right| .
$$




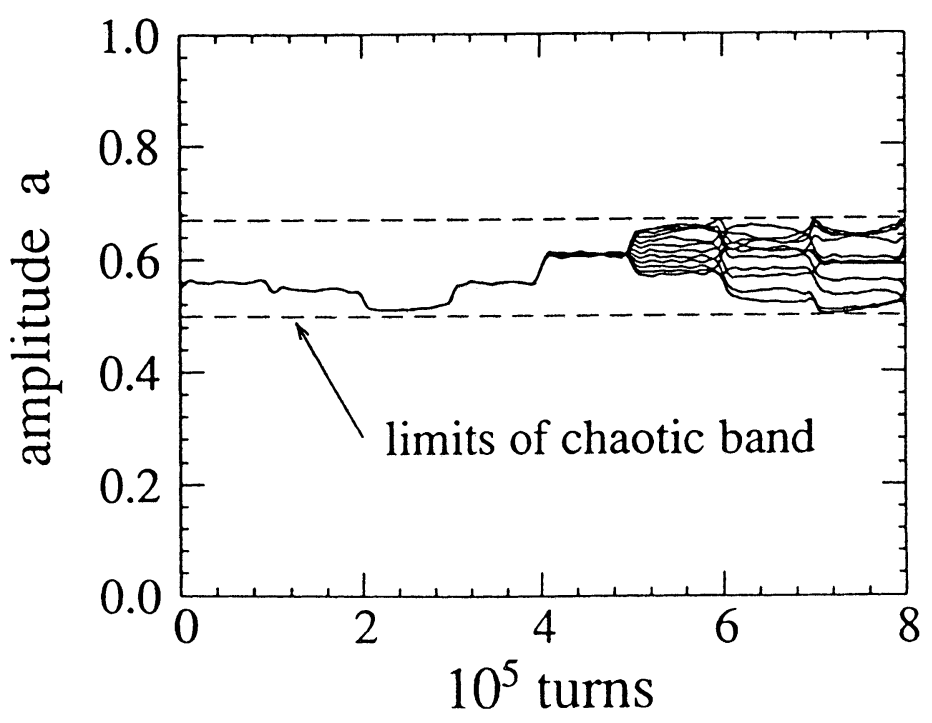

FIGURE 8a: The chaotic band created by the overlap of nine static islands is shown by following twelve trajectories with initial conditions that differ by $1 \times 10^{-6}$ in initial amplitude.

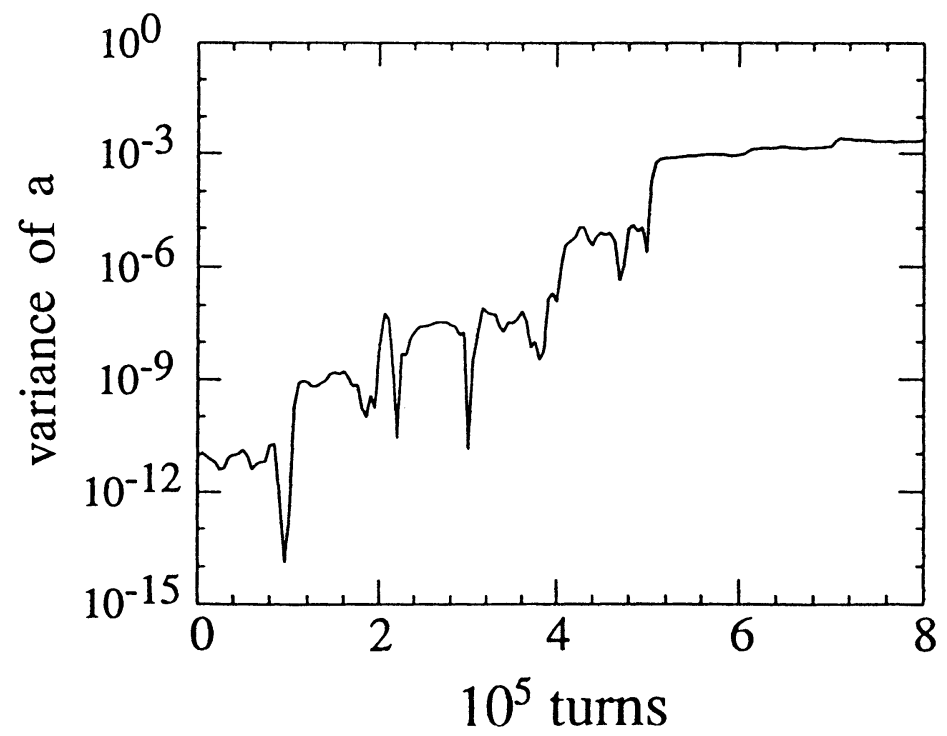

FIGURE 8b: The variance of the amplitude of the twelve particles in the chaotic band as a function of time. The approximately linear growth trend in the log-lin plot indicates exponential trajectory divergence that is characteristic of chaotic behavior. 
Comparison with Equations (21) and (32b) shows that the condition for chaotic overlap is simply

$$
\left|Q_{1}-Q_{2}\right|<2 \varepsilon^{1 / 2} a Q_{s 0} .
$$

This condition is readily extended to a system of several resonance islands.

Even in the presence of several discrete frequencies, the perturbation is deterministically described as the sum of sinusoids, so that the resulting chaotic motion is not true "noise" in the sense of being due to a random or unknown source. Nor is the chaotic motion truly diffusive, in the sense of phase-space deviations evolving proportional to the square root of time. In fact, a characteristic signature of chaotic motion is that two trajectories starting off infinitesimally close together in phase space diverge exponentially as a function of time. The exponent describing this behavior is known as the Lyapunov exponent. Figures $8 \mathrm{a}$ and $8 \mathrm{~b}$ illustrate this aspect of the motion, using results from a simulation of a chaotic band in the Tevatron from amplitudes of about 0.50 to 0.67 caused by nine overlapping resonance islands of strength $\varepsilon=0.0007$, much weaker than the nominal drive-resonance strength. The amplitude of twelve neighboring protons is plotted versus time in Figure 8a, showing explosive divergence due to the chaotic nature of the motion. Figure $8 \mathrm{~b}$ verifies that the motion is indeed chaotic by showing that the variance of the amplitude distribution grows exponentially over 8 orders of magnitude until the trajectories fill the entire width of the chaotic band. This is in marked contrast to a white-noise process, in which the variance of a distribution grows linearly in time.

Figure 8 also shows that it takes at least 500,000 turns ( 500 synchrotron periods, 10 seconds in the Tevatron, 2.5 minutes in the SSC) for protons to move from the bottom of the chaotic band up to the top. A rough estimate for this transit time goes as follows. It takes about $\Delta t \approx 1 /\left(2 Q_{I}\right)$ turns to move from the bottom to the top of an individual island. Therefore the minimum time to propagate across the 9-island chaotic band is $\Delta t \approx 9 /\left(a_{r} Q_{s 0} \varepsilon^{1 / 2}\right)=575 T_{s}$, in good agreement with the simulation. This time scale is appropriate for crystal extraction purposes, as it is much less than typical beamstorage times of about a day. It is also larger than or comparable to the time needed for islands to adiabatically move from the beam core to the drive islands, showing that a chaotic band may be used as a convenient pulse stretcher. Since the number of islands necessary to cause chaos across a given range of amplitudes is inversely proportional to $\varepsilon^{1 / 2}$, the time for band crossing scales inversely with $\varepsilon$, offering a convenient way to adjust the length of the pulse.

\section{A PROTOTYPICAL INTEGRATED EXTRACTION SCHEME}

It is interesting to envisage and simulate a plausible crystal extraction scheme that uses all three voltage modulation mechanisms described above. Figure 9 illustrates the response of a test particle to the scheme. A feed resonance is used to trap the proton at an amplitude of about $a \approx 0.2$, and then to adiabatically transport it for about $2,000,000$ turns ( 10 minutes in the SSC), until, at an amplitude of about $a \approx 0.50$, 


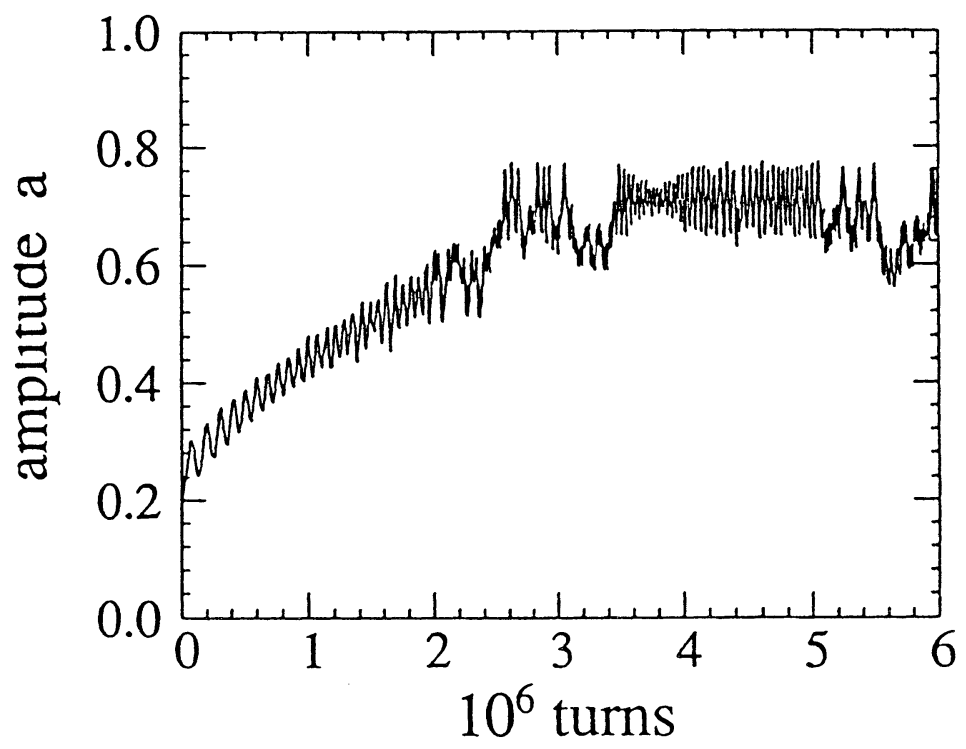

FIGURE 9: A single trajectory is followed for a system excited by seven discrete amplitude modulation sinusoids. The tune of one of the sinusoids is ramped to provide a moving "feed" island. Five more are static, and create resonance islands that overlap to form a chaotic layer. The seventh perturbation is strong, and creates a "drive" island at a large amplitude.

it enters a chaotic band made up of five overlapping resonances. The proton then takes about another 500,000 turns to "diffuse" across to the outside of the chaotic band, at about $a \approx 0.64$, where the chaotic band touches the drive resonance. The drive resonance is not destroyed if its width is much larger than the islands of the chaotic band - the small overlap merely creates a "hole" in the drive "bucket". After entering the drive island, which extends up to an amplitude of about $a \approx 0.78$, the proton is rapidly accelerated into a crystal that is imagined to be placed at about $a \approx 0.71$. Since the crystal is not included in the simulation model, the proton is subsequently able to escape back into the chaotic band. The proton continues to re-enter and re-exit the drive resonance at irregular later times, showing that there is a considerable spread in the amount of time that protons take to get from the chaotic band into the crystal. Given the right parameters, the scheme will provide a slow, steady spill.

The motion of a single particle in Figure 9 indicates that the scheme has many of the desired characteristics for a crystal channeling extraction system. Figures $10 \mathrm{a}$ and $10 \mathrm{~b}$ show results from a more-sophisticated simulation of the probable extraction efficiency, in which the distribution of the penetration depth into the crystal is measured for a statistical distribution of many test protons. The phase-space trajectory of each individual proton is followed as it is injected at the low-amplitude edge of the chaotic layer, diffuses up through the layer, is captured by the drive resonance, and finally 


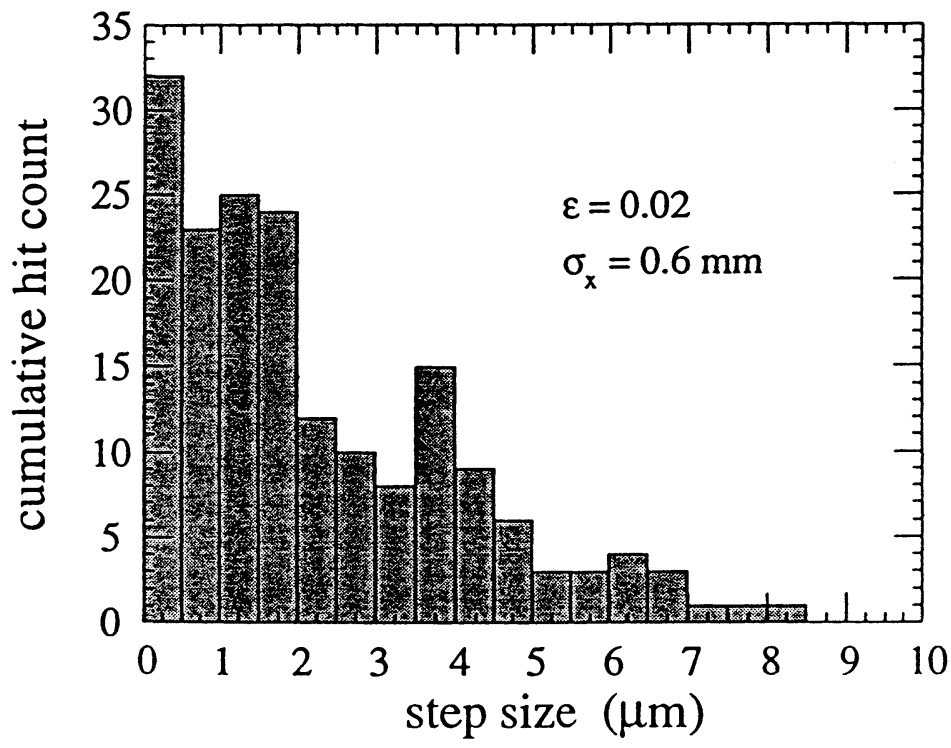

FIGURE 10a: Distribution of the depth of proton penetrations, as predicted for the Tevatron crystal channeling extraction experiment, using the parameters mentioned in the text.

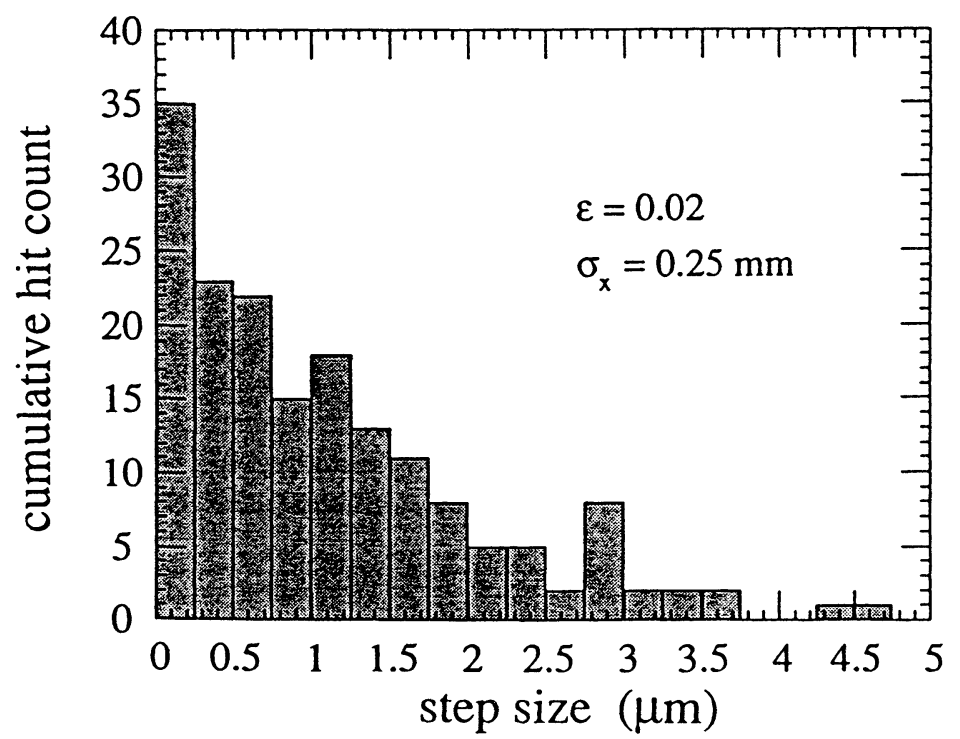

FIGURE 10b: Depth of proton penetrations distribution on the crystal for the SFT fixed target B-physics experiment at the SSC, using preliminary parameters mentioned in the text. 
is taken to large amplitudes, where it hits the crystal. After each hit the penetration depth is recorded and a new proton is launched. The protons have random initial betatron and synchrotron phases and a Gaussian distribution of betatron amplitudes. The drive resonance is powered by a modulation of amplitude $\varepsilon=0.02$ at a tune of $Q_{m}=0.86 Q_{s 0}$, and the five overlapping resonances are due to modulations of amplitude $\varepsilon=0.002$ at $Q_{m} / 2 Q_{s 0}=0.905,0.915,0.925,0.935$ and 0.945 . For the Tevatron case the fractional horizontal tune is taken to be 0.413 , and the crystal edge is $1.8 \mathrm{~mm}$ off axis. The same tune is used for the SSC case, but the crystal edge is placed at $0.95 \mathrm{~mm}$. All other relevant parameters can be found in Table 1 .

For the Tevatron experiment, Figure 10a, the maximum step size is more than $8 \mu \mathrm{m}$, and the median step size is $2 \mu \mathrm{m}$. For the SFT extraction system at the SSC, Figure 10b, the maximum step size is over $4.5 \mu \mathrm{m}$, and the median step size is about $1 \mu \mathrm{m}$. These numbers are acceptable in both cases, but are smaller than in the simple theoretical analysis given above for a variety of reasons. One of the most important effects is that the proton enters the drive resonance near its separatrix. The island tune is decreased in this region of the resonance, in the same way that large amplitude trajectories in the main rf bucket have tunes smaller than $Q_{s 0}$. Thus the synchrotron amplitude growth rate is smaller than our estimate, which employed the small-amplitude island tune. Also, the spread in betatron amplitudes gives a spread in synchrotron amplitudes at which the proton strikes the crystal, again causing a degradation in the step size due to the synchrotron amplitude growth rate being smaller than the previous estimate.

It should be noted that the parameters assumed for the simulations have been not well optimized. The fact that acceptable step size distributions are generated even by these preliminary calculations is encouraging, as the distributions can undoubtedly be improved. The simulations suggest that a good rule of thumb is that the median step size is an order of magnitude smaller than one would estimate from the quantity $g a_{s}$.

\section{CONCLUSIONS}

The underlying behavior of a prototypical scheme based on amplitude modulation at a small number of discrete frequencies has been analyzed, and is quantitatively understood in terms of a relatively small number of well-established accelerator physics concepts. Simulation tests of the quantitative descriptions of these concepts have all been successful.

Three mechanisms have been identified as potentially relevant to a crystal channeling extraction system: drive extraction by a strong stationary island, adiabatic transport by a feed island, and "diffusive" transport by chaotic overlap. The modulation frequencies involved in all of these mechanisms are low - of order $100 \mathrm{~Hz}$. While the efficiency of all three mechanisms depends on accurate knowledge of the smallamplitude synchrotron frequency $Q_{s 0}$ at the $1 \%$ level, this is not hard to achieve in practice.

A prototypical scheme that uses all three rf mechanisms has been demonstrated to work well, by theoretical analysis and by simulation. Typical step sizes of 5-10 $\mu \mathrm{m}$ are predicted, more than adequate for efficient channeling. 
Three times that are characteristic of the extraction process need to be commensurate for efficient extraction. This is true for all rf-manipulation extraction schemes (including noise-based schemes) which ultimately use spiral motion in longitudinal phase space.

A minor demand is made on the horizontal betatron tune in the extraction scheme - it should not be set near any low order resonance. This is also desirable in practical collider operation for other reasons. Extraction schemes based on resonance excitation in horizontal phase space require the opposite condition.

Can bent-crystal extraction for fixed-target beauty physics be efficient, and be parasitic to collider operation of the SSC? The preliminary conclusion here, from the point of view of a paper study of longitudinal dynamics, is "yes."

\section{ACKNOWLEDGMENTS}

We would like to express our sincere thanks to Thornton Murphy for the friendly interest, encouragement, and comments that he never failed to provide during the work that led to the writing of this paper.

\section{REFERENCES}

1. B. Cox et al., "The possibility of parasitic $20 \mathrm{TeV}$ beams extracted from the SSC using bent crystals," in Proceedings of the 1988 Summer Study on the Design and Utilization of the SSC (Snowmass CO, 1988), p. 536.

2. The SFT Collaboration, "An expression of interest in a super fixed target beauty facility at the SSC," document, EOI-14, submitted to the SSC PAC program Advisory Committee (1990).

3. H. Brown et al., "Report of the Super Fixed Target Beauty Facility Working Group on Progress towards the SFT at the SSC," in Proceedings of the 1990 Summer Study on the Physics of the SSC (Snowmass CO, 1990).

4. M. Harrison, "Resonant extraction at the Tevatron," in Physics of Particle Accelerators, AIP Conference Proceedings 184, edited by M. Month and M. Dienes (AIP, New York, 1989), Vol. 2, p. 2009.

5. S. Peggs, "Proton mining — dual frequency amplitude modulation," Fermilab internal note AP 91001 (1991).

6. B. Newberger and H. Shih, "Low intensity beam extraction at the SSC," SSC Laboratory internal note SSCL-344 (1991).

7. H. Shih and A. Taratin, "Bent crystal extraction of the SSC beam with rf noise induced diffusion," SSC Laboratory internal note SSCL-389 (1991).

8. J. Ellison, B. Newberger, and H. Shih, "Effect of if noise on the SSC beam," in Proceedings of the IEEE Particle Accelerator Conference (San Francisco, CA 1991), p. 216.

9. For an excellent general reference paper, see G. Dome, "Theory of $\mathrm{rf}$ acceleration and if noise," CERN report 84-15 (1984).

10. C.T. Murphy et al., "Proposal for a test of low intensity extraction from the Tevatron using channeling in a bent crystal," proposal P-853, submitted to the Fermilab Program Advisory Committee (1991).

11. N. McLachlan, Theory and Application of Mathieu Functions (Oxford University Press, 1947).

12. S. Peggs, "Hamiltonian theory of the E778 Experiment," in Proceedings of the Second ICFA Beam Dynamics Workshop (Lugano, Switzerland, 1988). Also published as SSC Laboratory internal note SSC-175 (1988). 